UNIVERSITÀ DEGLI STUDI DI BERGAMO

DIPARTIMENTO DI INGEGNERIA DELL'INFORMAZIONE

E METODI MATEMATICI

QUADERNI DEL DIPARTIMENTO

Department of Information Technology and Mathematical Methods

Working Paper

Series "Mathematics and Statistics"

n. $11 / \mathrm{MS}-2012$

Optimized Schwarz methods for the

diffusion-reaction problem with cylindrical interfaces

by

G. Gigante, M. Pozzoli, C. Vergara 


\section{COMITATO DI REDAZIONE ${ }^{\S}$}

Series Information Technology (IT): Stefano Paraboschi

Series Mathematics and Statistics (MS): Luca Brandolini, Ilia Negri

\footnotetext{
$\S$ L'accesso alle Series è approvato dal Comitato di Redazione. I Working Papers della Collana dei Quaderni del Dipartimento di Ingegneria dell'Informazione e Metodi Matematici costituiscono un servizio atto a fornire la tempestiva divulgazione dei risultati dell'attività di ricerca, siano essi in forma provvisoria o definitiva.
} 


\title{
OPTIMIZED SCHWARZ METHODS FOR THE DIFFUSION-REACTION PROBLEM WITH CYLINDRICAL INTERFACES *
}

\author{
GIACOMO GIGANTE ${ }^{\dagger}$, MATTEO POZZOLI $^{\ddagger}$, AND CHRISTIAN VERGARA $§$
}

\begin{abstract}
In this work we consider the Optimized Schwarz Method for the three-dimensional (3D) diffusion-reaction problem. In particular, we treat the case of cylindrical interfaces between the subdomains, and we provide for such case a convergence analysis of the Schwarz method, both in the case of Dirichlet interface conditions and in that of general transmission conditions. This allows to recover, for the latter case, optimal symbols for the interface conditions, which are supposed to work well for geometries which feature cylindrical interfaces. Moreover, starting from these optimal symbols, we propose effective and easily computable constant interface parameters, to be used in the numerical simulations. We finally present several 3D numerical results aiming at validating the theoretical findings.
\end{abstract}

Key words. Optimized Schwarz Method, Robin interface conditions, cylindrical coordinates, Fourier transform.

AMS subject classifications. $65 \mathrm{~N} 12,65 \mathrm{~N} 30,42 \mathrm{~B} 37$

1. Introduction. The classical Schwarz method for the numerical solution of a partial differential equation consists in the splitting of the computational domain in two (or even more) subdomains, with or without overlap, and in the solution of the equation at hand in such subdomains in an iterative framework, through the exchange at the interface of the trace of the solution. It is known that this method features a slow convergence in general and does not converge without overlap [4,22, 24], unless a non-conforming discretization is employed $[2,3,7]$. For this reason, Lions introduced different transmission conditions, of Robin type, which allowed to improve the convergence properties and to reach converge also without overlap [16]. Successively, this method has been generalized by considering general, more performing interface conditions, involving non-local transmission operators (generalized Schwarz method, see, e.g., $[5,19])$.

The choice of suitable parameters in such operators is crucial to guarantee good convergence properties. This is usually driven by the minimization of the reduction factor related to the iterations (Optimized Schwarz Method). To obtain the reduction factor usually the Fourier transform is applied to some variables leading to ordinary differential equations [13]. This optimization strategy has been applied to a great variety of problems. We cite, for example, the advection-reaction-diffusion problem $[8,14]$, the Helmholtz equation $[11,18]$, the coupling of heterogeneous media $[9,10$, 17], the shallow-water equations [21], the Maxwell's equations [6], the fluid-structure interaction (FSI) problem [12] and the scattering problem [23].

* This work has been partially supported by the ERC Advanced Grant N.227058 MATHCARD and by the Italian MIUR PRIN09 project n. 2009Y4RC3B_001. The numerical simulations have been performed at CILEA Consortium through a LISA Initiative (Laboratory for Interdisciplinary Advanced Simulation) 2012 grant [link:http://lisa.cilea.it].

${ }^{\dagger}$ Dipartimento di Ingegneria dell'Informazione e Metodi Matematici, Università degli Studi Bergamo, Italy, (giacomo.gigante@unibg.it)

¥Dipartimento di Ingegneria dell’Informazione e Metodi Matematici, Università degli Studi Bergamo, Italy, (matteo.pozzoli@unibg.it)

$\S$ Dipartimento di Ingegneria dell'Informazione e Metodi Matematici, Università degli Studi Bergamo, Italy, (christian.vergara@unibg.it) 
The convergence and optimization analyses performed in such works have been obtained mainly for two-dimensional (2D) domains. In [6] a three-dimensional (3D) optimization procedure has been proposed for the Maxwell's equations. In any case, to the best of the authors' knowledge, all these analyses have been performed for flat interfaces among subdomains. Although the optimized parameters derived from such optimization procedures have been shown to be effective in some context also for numerical simulations with curve interfaces (see for example [20] for FSI), a 3D optimization procedure which exploits the particular configuration of the computational domain could allow to compute more efficient parameters, speeding up the convergence of the numerical scheme.

The goal of this paper is to perform, for the diffusion-reaction problem, new $3 \mathrm{D}$ convergence analyses and optimization procedures for parameters involved in the transmission conditions. We firstly consider the case of the whole space $\mathbb{R}^{3}$ divided in two overlapping subdomains, with the overlapping region delimited by two flat parallel surfaces (used in [6] for the Maxwell's equations). For this reason, we refer to these cases as "flat" analysis and "flat" optimization procedure. Then, we move towards a different case, where the interfaces delimiting the overlapping region are curve and the problem features a cylindrical symmetry. We perform for this case a convergence analysis of the classical and of the generalized Schwarz methods ("cylindrical" analysis), which show that without overlap the classical Schwarz method does not converge, while the generalized Schwarz method converges. Moreover, such analyses show that in the case with overlap, the generalized Schwarz method converges faster than the classical one, provided that the symbols in the transmission conditions are suitably chosen.

For the generalized Schwarz method, we look also for optimal values of the parameters involved in the transmission conditions (flat and cylindrical optimizations). The hope is to find optimal parameters which could be effective for problems solved in domains characterized by a cylindrical symmetry. The optimization procedures show that in the "flat" case the same parameters of the 2D analysis are recovered. Viceversa, for curve interfaces the optimization procedure leads to new optimized parameters, which are supposed to work better in presence of a cylindrical symmetry. To prove this, we performed several numerical experiments which confirmed the theoretical findings.

The outline of the paper is as follows. In Section 2 we present the 3D "flat" optimization procedure. In Section 3.1 we show the 3D convergence analysis of the classical Schwarz method for the case of cylindrical interfaces, while in Section 3.2 we do the same for the generalized Schwarz algorithm. In Section 4 we perform the optimization procedure based on the minimization of the reduction factor found in Section 3.2. Finally, in Section 5 we show the numerical results obtained by 3D numerical experiments.

2. A flat 3D convergence analysis and optimization. In this paper, we consider the diffusion-reaction model problem

$$
-\triangle u+\eta u=f \quad x \in \Omega \equiv \mathbb{R}^{3}, \eta>0,
$$

for a given function $f$ and where the solution $u$ is required to be continuous and to decay at infinity.

In this section we consider $\Omega$ subdivided into the two overlapping subdomains [6]

$$
\Omega_{1}=(-\infty, L) \times \mathbb{R}^{2}, \quad \Omega_{2}=(0, \infty) \times \mathbb{R}^{2},
$$


for $L \geq 0$, and we consider the 3D extension of the 2D analyses performed so far in the context of the Optimized Schwarz Methods [8]. We consider directly the generalized Schwarz method, where the transmission interface conditions are of type

$$
\left(\mathcal{S}_{j}+\frac{\partial}{\partial x}\right) u_{j}(\widetilde{x}, y, z)=P_{j}(\widetilde{x}, y, z), j=1,2,
$$

for suitable linear operators $\mathcal{S}_{j}, j=1,2$, along the interfaces $x=\widetilde{x}$, with $\widetilde{x}=L$ and $\widetilde{x}=0$, respectively, and suitable right hand sides $P_{j}, j=1,2$. Then, the generalized Gauss-Seidel-Schwarz method at iteration $n$ reads as follows

$$
\begin{aligned}
& \begin{cases}(\eta-\Delta) u_{1}^{n}=f & \text { in } \Omega_{1}, \\
\left(\mathcal{S}_{1}+\frac{\partial}{\partial x}\right) u_{1}^{n}(L, y, z)=\left(\mathcal{S}_{1}+\frac{\partial}{\partial x}\right) u_{2}^{n-1}(L, y, z) & (y, z) \in \mathbb{R}^{2},\end{cases} \\
& \begin{cases}(\eta-\Delta) u_{2}^{n}=f & \text { in } \Omega_{2}, \\
\left(\mathcal{S}_{2}+\frac{\partial}{\partial x}\right) u_{2}^{n}(0, y, z)=\left(\mathcal{S}_{2}+\frac{\partial}{\partial x}\right) u_{1}^{n}(0, y, z) & (y, z) \in \mathbb{R}^{2},\end{cases}
\end{aligned}
$$

and we require the iterates to decay at infinity.

The convergence analysis of the previous algorithm is performed in the homogeneous case due to the linearity of the problem. Therefore, we set, here and elsewhere, $f=0$. The analysis is based on the Fourier transform $\mathcal{F}$ in the variables $y$ and $z$,

$$
\widehat{g}(x, s, k)=\mathcal{F}(g):=\int_{-\infty}^{+\infty} \int_{-\infty}^{+\infty} g(x, y, z) e^{-i s y} e^{-i k z} d y d z
$$

where $s, k \in \mathbb{R}$ are the coordinates in the frequency domain.

We have the following result.

Proposition 1. The reduction factor $\rho^{\text {flat }}$ related to iterations $(2.2)$ is given by

$$
\rho^{f l a t}(s, k)=\frac{\left(\sigma_{1}-\beta\right)\left(\sigma_{2}+\beta\right)}{\left(\sigma_{1}+\beta\right)\left(\sigma_{2}-\beta\right)} e^{-2 \beta L},
$$

where $\beta=\sqrt{\left(s^{2}+k^{2}\right)+\eta}$ and $\sigma_{j}(s, k), j=1,2$, denote the symbols of $\mathcal{S}_{j}, j=1,2$.

Proof. Applying the Fourier transform (2.3) to iterations (2.2), we obtain

$$
\begin{aligned}
& \left\{\begin{array}{lc}
\beta^{2}{\widehat{u_{1}}}^{n}-\frac{\partial^{2}}{\partial x^{2}}{\widehat{u_{1}}}^{n}=0 & \text { in } \Omega_{1}, \\
\left(\sigma_{1}+\frac{\partial}{\partial x}\right){\widehat{u_{1}}}^{n}(L, s, k)=\left(\sigma_{1}+\frac{\partial}{\partial x}\right){\widehat{u_{2}}}^{n-1}(L, s, k) & (s, k) \in \mathbb{R}^{2},
\end{array}\right. \\
& \begin{cases}\beta^{2}{\widehat{u_{2}}}^{n}-\frac{\partial^{2}}{\partial x^{2}}{\widehat{u_{2}}}^{n}=0 & \text { in } \Omega_{2}, \\
\left(\sigma_{2}+\frac{\partial}{\partial x}\right){\widehat{u_{2}}}^{n}(0, s, k)=\left(\sigma_{2}+\frac{\partial}{\partial x}\right){\widehat{u_{2}}}^{n}(0, s, k) & (s, k) \in \mathbb{R}^{2} .\end{cases}
\end{aligned}
$$

The solutions of these ordinary differential equations (ODE's) in the variable $x$ are of type

$$
A e^{\beta x}+B e^{-\beta x} .
$$

Now, by using the conditions at infinity, the transmission conditions and by proceeding as in the $2 \mathrm{D}$ case (see, e.g., [8]), we obtain precisely (2.4).

We observe that the expression of the reduction factor (2.4) has exactly the same expression as in the $2 \mathrm{D}$ case, where $k^{2}$ is replaced by $s^{2}+k^{2}$. This means that when 

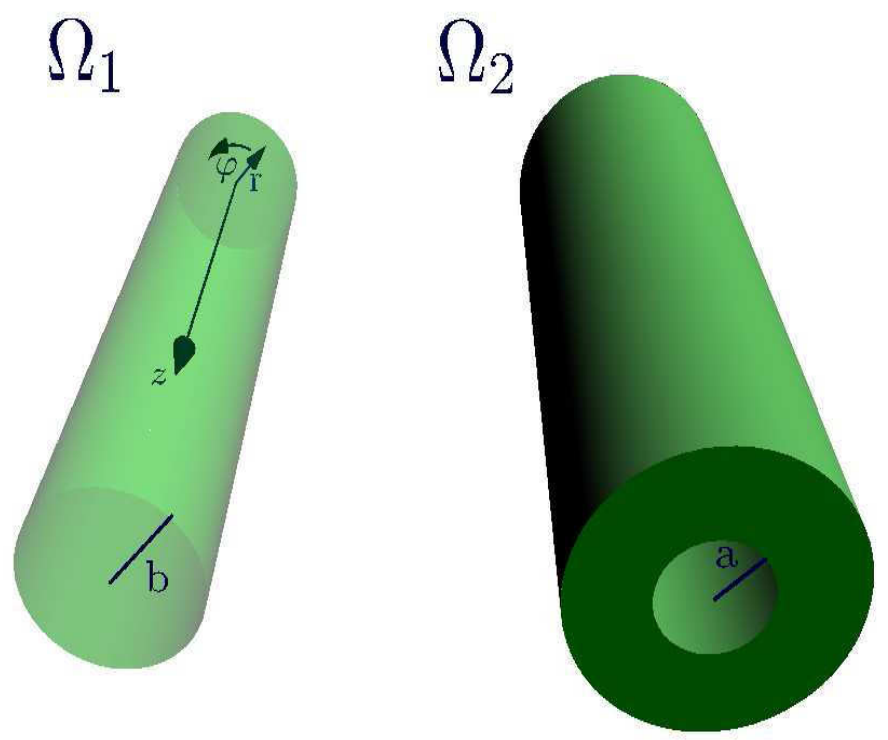

FIG. 3.1. Computational overlapping subdomains $\Omega_{1}$ and $\Omega_{2}$.

the interface is flat, the usual optimized choices of the symbols $\sigma_{1}$ and $\sigma_{2}$ derived by a $2 \mathrm{D}$ analysis [8] should work just as well in the $3 \mathrm{D}$ case as they do in the $2 \mathrm{D}$ case. In particular, in 3D the optimal values are

$$
\sigma_{1, \text { opt }}^{\text {flat }}=\sqrt{\left(s^{2}+k^{2}\right)+\eta}, \quad \sigma_{2, \text { opt }}^{\text {flat }}=-\sqrt{\left(s^{2}+k^{2}\right)+\eta},
$$

which annihilate $\rho^{\text {flat }}$ independently of the overlap $L$ and of the frequencies $s$ and $k$.

3. Convergence of the Schwarz algorithm for cylindrical interfaces. Due to the result of the previous section, it is reasonable to ask whether an optimization procedure related to different interface configurations could lead to different optimal values of $\sigma_{j}, j=1,2$.

With this aim, in this section we discuss the case where the interfaces are concentric cylindrical surfaces and we exploit the cylindrical symmetry (see Figure 3.1). Therefore, we consider again problem (2.1), but $\Omega$ in this case is decomposed into the two overlapping subdomains

$$
\Omega_{1}:=\{r<b, \varphi \in[0,2 \pi), z \in \mathbb{R}\}, \quad \Omega_{2}:=\{r>a, \varphi \in[0,2 \pi), z \in \mathbb{R}\},
$$

where $0<a \leq b$ are two given scalars. Due to the cylindrical symmetry of the subdomains, we have introduced the cylindrical coordinates $(r, \varphi, z)$, representing the distance from the $z$-axis, the angle and the height, respectively, and such that

$$
\left\{\begin{array}{l}
x=r \cos \varphi \\
y=r \sin \varphi \\
z=z,
\end{array}\right.
$$

(see Figure 3.1). We then rewrite the Laplacian with respect to these coordinates as follows

$$
\Delta_{c y l}=\frac{1}{r} \frac{\partial}{\partial r}\left(r \frac{\partial}{\partial r}\right)+\frac{1}{r} \frac{\partial^{2}}{\partial \varphi^{2}}+\frac{\partial^{2}}{\partial z^{2}} .
$$


In the next subsection, we discuss the case of the classical Schwarz method, while in subsection 3.2 we discuss the generalized Schwarz method.

3.1. The classical Schwarz algorithm. The classical Gauss-Seidel-Schwarz method for the problem at hand at iteration $n$ is given by

$$
\begin{aligned}
& \begin{cases}\left(\eta-\Delta_{c y l}\right) u_{1}^{n}=f & \text { in } \Omega_{1}, \\
u_{1}^{n}(b, \varphi, z)=u_{2}^{n-1}(b, \varphi, z) & (\varphi, z) \in[0,2 \pi) \times \mathbb{R}, \\
\int_{-\infty}^{\infty} \int_{0}^{2 \pi}\left|u_{1}^{n}(r, \varphi, z)\right| d \varphi d z \text { bounded } & \text { as } r \rightarrow 0^{+}, \\
u_{1}^{n}=0 & \text { in }\{z= \pm \infty, r \leq b\} ;\end{cases} \\
& \begin{cases}\left(\eta-\Delta_{c y l}\right) u_{2}^{n}=f & \text { in } \Omega_{2}, \\
u_{2}^{n}(a, \varphi, z)=u_{1}^{n}(a, \varphi, z) & (\varphi, z) \in[0,2 \pi) \times \mathbb{R}, \\
u_{2}^{n}=0 & \text { in }\{r=+\infty\} \cup\{z= \pm \infty, r \geq a\} .\end{cases}
\end{aligned}
$$

The analysis is based on the Fourier transform $\mathcal{F}^{c y l}$ in the cylindrical variables $\varphi$ and $z$, given by

$$
\widehat{g}(r, m, k)=\mathcal{F}^{c y l}(g):=\int_{-\infty}^{+\infty} \int_{0}^{2 \pi} g(r, \varphi, z) e^{-i m \varphi} d \varphi e^{-i z k} d z,
$$

where $m \in \mathbb{Z}$ and $k \in \mathbb{R}$ are the coordinates in the frequency domain. We observe that hypothesis $(3.1)_{3}$ has been made in order to guarantee that the Fourier transform $\widehat{u}_{1}$ is bounded for any $m$ and $k$ as $r \rightarrow 0^{+}$.

We have the following result.

Proposition 2. The reduction factor $\rho_{\text {cla }}^{c y l}$ related to iterations $(3.1)$ is given by

$$
\rho_{c l a}^{c y l}(m, k)=\frac{I_{m}(\alpha a)}{I_{m}(\alpha b)} \frac{K_{m}(\alpha b)}{K_{m}(\alpha a)},
$$

where

$$
\alpha=\sqrt{k^{2}+\eta}
$$

and $I_{m}$ and $K_{m}$ are the Bessel functions of imaginary argument (modified Bessel functions, see the Appendix).

Proof. Applying the Fourier transform (3.2) to iterations (3.1), we obtain the following ODE's

$$
\eta{\widehat{u_{j}}}^{n}-\frac{1}{r} \frac{\partial}{\partial r}\left(r \frac{\partial{\widehat{u_{j}}}^{n}}{\partial r}\right)+\frac{1}{r^{2}} m^{2}{\widehat{u_{j}}}^{n}+k^{2}{\widehat{u_{j}}}^{n}=0 \quad j=1,2,
$$

where $\widehat{u_{j}}=\widehat{u_{j}}(r, m, k), j=1,2$, are the Fourier transforms of $u_{j}(r, \varphi, z), j=1,2$, in the variables $\varphi, z$ as in (3.2). Therefore, each of the two equations in (3.1) becomes

$$
\left(r^{2} \frac{\partial^{2}}{\partial r^{2}}+r \frac{\partial}{\partial r}-\left(m^{2}+r^{2}\left(k^{2}+\eta\right)\right)\right) \widehat{u}_{j}^{n}=0 .
$$

These are essentially modified Bessel equations (see (5.1)), whose solutions in $(0,+\infty)$ are

$$
A I_{m}(\alpha r)+B K_{m}(\alpha r)
$$


for suitable coefficients $A$ and $B$ and where $\alpha$ is given by (3.4).

Our assumptions on the behaviour of $u_{1}^{n}(r, \varphi, z)$ as $r \rightarrow 0^{+}$and of $u_{2}^{n}(r, \varphi, z)$ as $r \rightarrow+\infty$, and the monotonicity of the modified Bessel functions (see Figure 5.4), give

$$
\begin{aligned}
& {\widehat{u_{1}}}^{n}(r, m, k)=A_{n}(m, k) I_{m}(\alpha r), \\
& {\widehat{u_{2}}}^{n}(r, m, k)=B_{n}(m, k) K_{m}(\alpha r),
\end{aligned}
$$

for suitable functions $A_{n}$ and $B_{n}$, which are determined by the interface conditions

$$
\begin{aligned}
& {\widehat{u_{1}}}^{n}(b, \cdot, \cdot)={\widehat{u_{2}}}^{n-1}(b, \cdot, \cdot), \\
& {\widehat{u_{2}}}^{n}(a, \cdot, \cdot)={\widehat{u_{1}}}^{n}(a, \cdot, \cdot) .
\end{aligned}
$$

From the first of (3.5) we obtain

$$
A_{n}(m, k) I_{m}(\alpha b)={\widehat{u_{2}}}^{n-1}(b, m, k) .
$$

Therefore, we have

$$
A_{n}(m, k)=\frac{{\widehat{u_{2}}}^{n-1}(b, m, k)}{I_{m}(\alpha b)},
$$

and the solution ${\widehat{u_{1}}}^{n}$ is then given by

$$
{\widehat{u_{1}}}^{n}(r, m, k)=\frac{I_{m}(\alpha r)}{I_{m}(\alpha b)}{\widehat{u_{2}}}^{n-1}(b, m, k) .
$$

Analogously, we obtain

$$
{\widehat{u_{2}}}^{n}(r, m, k)=\frac{K_{m}(\alpha r)}{K_{m}(\alpha a)}{\widehat{u_{1}}}^{n}(a, m, k) .
$$

Therefore, by evaluating ${\widehat{u_{1}}}^{n}(r, m, k)$ for $r=a$ and ${\widehat{u_{2}}}^{n}(r, m, k)$ for $r=b$, we obtain

$$
{\widehat{u_{2}}}^{n}(b, m, k)=\frac{I_{m}(\alpha a)}{I_{m}(\alpha b)} \frac{K_{m}(\alpha b)}{K_{m}(\alpha a)}{\widehat{u_{2}}}^{n-1}(b, m, k),
$$

which concludes the proof.

REMARK 1. In the case of a vanishing overlap $(a=b)$, from (3.3) we obtain $\rho_{c l a}^{c y l}=1$, that is the classical Schwarz method does not converge. Viceversa, by exploiting the properties of the modified Bessel functions (see Figure 5.4), for $a<b$ we obtain $I_{m}(a)<I_{m}(b)$ and $K_{m}(a)>K_{m}(b), \forall m$, so that $\rho_{\text {cla }}^{\text {cyl }}<1$. Therefore, the classical Schwarz method converges in presence of an overlap, and the convergence is faster for increasing overlaps. These observations confirm qualitatively the convergence properties of the classical Schwarz algorithm for the "flat" case.

3.2. The generalized Schwarz algorithm. We consider here the generalized Gauss-Seidel-Schwarz algorithm, given by

$$
\begin{aligned}
& \begin{cases}\left(\eta-\Delta_{c y l}\right) u_{1}^{n}=f & \text { in } \Omega_{1}, \\
\left(\mathcal{S}_{1}+\frac{\partial}{\partial r}\right) u_{1}^{n}(b, \varphi, z)=\left(\mathcal{S}_{1}+\frac{\partial}{\partial r}\right) u_{2}^{n-1}(b, \varphi, z) & (\varphi, z) \in[0,2 \pi) \times \mathbb{R}, \\
\int_{-\infty}^{\infty} \int_{0}^{2 \pi}\left|u_{1}^{n}(r, \varphi, z)\right| d \varphi d z \text { bounded } & \text { as } r \rightarrow 0^{+}, \\
u_{1}^{n}=0 & \text { in }\{z= \pm \infty, r \leq b\} ;\end{cases} \\
& \begin{cases}\left(\eta-\Delta_{c y l}\right) u_{2}^{n}=f & \text { in } \Omega_{2}, \\
\left(\mathcal{S}_{2}+\frac{\partial}{\partial r}\right) u_{2}^{n}(a, \varphi, z)=\left(\mathcal{S}_{2}+\frac{\partial}{\partial r}\right) u_{1}^{n}(a, \varphi, z) & (\varphi, z) \in[0,2 \pi) \times \mathbb{R}, \\
u_{2}^{n}=0 & \text { in }\{r=+\infty\} \cup\{z= \pm \infty, r \geq a\} .\end{cases}
\end{aligned}
$$


We have the following result.

Proposition 3. The reduction factor $\rho^{\text {cyl }}$ related to iterations (3.6) is given by

$$
\rho^{c y l}(m, k)=\frac{\mathcal{I}_{2}(a, m, k)}{\mathcal{K}_{2}(a, m, k)} \frac{\mathcal{K}_{1}(b, m, k)}{\mathcal{I}_{1}(b, m, k)},
$$

where

$$
\begin{aligned}
& \mathcal{I}_{1}(r, m, k):=\sigma_{1}(m, k) I_{m}(\alpha r)+\alpha I_{m}^{\prime}(\alpha r), \\
& \mathcal{I}_{2}(r, m, k):=\sigma_{2}(m, k) I_{m}(\alpha r)+\alpha I_{m}^{\prime}(\alpha r), \\
& \mathcal{K}_{1}(r, m, k):=\sigma_{1}(m, k) K_{m}(\alpha r)+\alpha K_{m}^{\prime}(\alpha r), \\
& \mathcal{K}_{2}(r, m, k):=\sigma_{2}(m, k) K_{m}(\alpha r)+\alpha K_{m}^{\prime}(\alpha r),
\end{aligned}
$$

and $\alpha$ is given by (3.4).

Proof. By applying the Fourier transform (3.2) to iterations (3.6) we obtain again solutions of type

$$
\begin{aligned}
& {\widehat{u_{1}}}^{n}(r, m, k)=A_{n}(m, k) I_{m}(\alpha r), \\
& {\widehat{u_{2}}}^{n}(r, m, k)=B_{n}(m, k) K_{m}(\alpha r),
\end{aligned}
$$

for suitable functions $A_{n}$ and $B_{n}$, which, in this case, are determined by the interface conditions

$$
\left\{\begin{array}{l}
\left(\sigma_{1}+\frac{\partial}{\partial r}\right){\widehat{u_{1}}}^{n}(b, \cdot, \cdot)=\left(\sigma_{1}+\frac{\partial}{\partial r}\right){\widehat{u_{2}}}^{n-1}(b, \cdot, \cdot), \\
\left(\sigma_{2}+\frac{\partial}{\partial r}\right){\widehat{u_{2}}}^{n}(a, \cdot, \cdot)=\left(\sigma_{2}+\frac{\partial}{\partial r}\right){\widehat{u_{1}}}^{n}(a, \cdot, \cdot) .
\end{array}\right.
$$

By observing that

$$
\frac{\partial{\widehat{u_{1}}}^{n}}{\partial r}=A_{n} \alpha I_{m}^{\prime}(\alpha r), \quad \frac{\partial{\widehat{u_{2}}}^{n}}{\partial r}=B_{n} \alpha K_{m}^{\prime}(\alpha r),
$$

we obtain from the interface condition $(3.8)_{1}$

$$
\sigma_{1} A_{n} I_{m}(\alpha b)+A_{n} \alpha I_{m}^{\prime}(\alpha b)=\sigma_{1} B_{n-1} K_{m}(\alpha b)+B_{n-1} \alpha K_{m}^{\prime}(\alpha b),
$$

and then

$$
A_{n}=\frac{\sigma_{1} K_{m}(\alpha b)+\alpha K_{m}^{\prime}(\alpha b)}{\sigma_{1} I_{m}(\alpha b)+\alpha I_{m}^{\prime}(\alpha b)} B_{n-1} .
$$

Analogously, from the second of (3.8), we obtain

$$
B_{n}=\frac{\sigma_{2} I_{m}(\alpha a)+\alpha I_{m}^{\prime}(\alpha a)}{\sigma_{2} K_{m}(\alpha a)+\alpha K_{m}^{\prime}(\alpha a)} A_{n},
$$

so that we find a recursive formula for $B_{n}$ which reads

$$
B_{n}=\rho^{c y l} B_{n-1},
$$

with $\rho^{c y l}$ given by (3.7). This concludes the proof. 
We have the following result, which is the counterpart for cylindrical interfaces of that in Theorem 4.1 in [8] for flat interfaces.

Proposition 4. The generalized Schwarz method (3.6) converges faster than the classical Schwarz method (3.1), provided that

$$
\begin{aligned}
& \sigma_{1}>-\frac{1}{2} \alpha\left(\frac{I_{m}^{\prime}(\alpha b)}{I_{m}(\alpha b)}+\frac{K_{m}^{\prime}(\alpha b)}{K_{m}(\alpha b)}\right), \\
& \sigma_{2}<-\frac{1}{2} \alpha\left(\frac{I_{m}^{\prime}(\alpha a)}{I_{m}(\alpha a)}+\frac{K_{m}^{\prime}(\alpha a)}{K_{m}(\alpha a)}\right) .
\end{aligned}
$$

Proof. The reduction factor (3.7) reads

$$
\begin{gathered}
\rho^{c y l}(m, k)=\frac{\sigma_{1} K_{m}(\alpha b)+\alpha K_{m}^{\prime}(\alpha b)}{\sigma_{1} I_{m}(\alpha b)+\alpha I_{m}^{\prime}(\alpha b)} \frac{\sigma_{2} I_{m}(\alpha a)+\alpha I_{m}^{\prime}(\alpha a)}{\sigma_{2} K_{m}(\alpha a)+\alpha K_{m}^{\prime}(\alpha a)}= \\
=\frac{\sigma_{1}+\frac{\alpha K_{m}^{\prime}(\alpha b)}{K_{m}(\alpha b)}}{\sigma_{1}+\frac{\alpha I_{m}^{\prime}(\alpha b)}{I_{m}(\alpha b)}} \frac{\sigma_{2}+\frac{\alpha I_{m}^{\prime}(\alpha a)}{I_{m}(\alpha a)}}{\sigma_{2}+\frac{\alpha K_{m}^{\prime}(\alpha a)}{K_{m}(\alpha a)}} \cdot \frac{K_{m}(\alpha b) I_{m}(\alpha a)}{I_{m}(\alpha b) K_{m}(\alpha a)}= \\
=\frac{\sigma_{1}+\frac{\alpha K_{m}^{\prime}(\alpha b)}{K_{m}(\alpha b)}}{\sigma_{1}+\frac{\alpha I_{m}^{\prime}(\alpha b)}{I_{m}(\alpha b)}} \frac{\sigma_{2}+\frac{\alpha I_{m}^{\prime}(\alpha a)}{I_{m}(\alpha a)}}{\sigma_{2}+\frac{\alpha K_{m}^{\prime}(\alpha a)}{K_{m}(\alpha a)}} \cdot \rho_{c l a}^{c y l}(m, k),
\end{gathered}
$$

where in the last equality we have exploited the definition of the convergence factor (3.3) of the classical Schwarz method. We have than to prove that

$$
\left|\frac{\sigma_{1}+\frac{\alpha K_{m}^{\prime}(\alpha b)}{K_{m}(\alpha b)}}{\sigma_{1}+\frac{\alpha I_{m}^{\prime}(\alpha b)}{I_{m}(\alpha b)}} \frac{\sigma_{2}+\frac{\alpha I_{m}^{\prime}(\alpha a)}{I_{m}(\alpha a)}}{\sigma_{2}+\frac{\alpha K_{m}^{\prime}(\alpha a)}{K_{m}(\alpha a)}}\right|<1
$$

for any $m, k$. Condition (3.10) follows for example if the two factors are both less than 1 , that is for

$$
\left|\frac{\sigma_{1}+\frac{\alpha K_{m}^{\prime}(\alpha b)}{K_{m}(\alpha b)}}{\sigma_{1}+\frac{\alpha I_{m}^{\prime}(\alpha b)}{I_{m}(\alpha b)}}\right|<1 \text { and } \quad\left|\frac{\sigma_{2}+\frac{\alpha I_{m}^{\prime}(\alpha a)}{I_{m}(\alpha a)}}{\sigma_{2}+\frac{\alpha K_{m}^{\prime}(\alpha a)}{K_{m}(\alpha a)}}\right|<1 .
$$

Since $-\frac{\alpha I_{m}^{\prime}(\alpha b)}{I_{m}(\alpha b)}<0<-\frac{\alpha K_{m}^{\prime}(\alpha b)}{K_{m}(\alpha b)}$, the first inequality follows when condition $(3.9)_{1}$ holds. Analogous arguments hold for the second factor in (3.10), which is less than 1 when condition $(3.9)_{2}$ holds. This concludes the proof.

4. 3D Optimization for cylindrical interfaces. From the expression of the reduction factor (3.7), we find that the optimal choices of $\sigma_{1}$ and $\sigma_{2}$ are those which annihilate $\mathcal{K}_{1}(b, m, k)$ and $\mathcal{I}_{2}(a, m, k)$, and therefore $\rho^{c y l}$, that is

$$
\begin{aligned}
\sigma_{1, o p t}^{c y l}(m, k) & =-\alpha \frac{K_{m}^{\prime}(\alpha b)}{K_{m}(\alpha b)}>0, \\
\sigma_{2, o p t}^{c y l}(m, k) & =-\alpha \frac{I_{m}^{\prime}(\alpha a)}{I_{m}(\alpha a)}<0 .
\end{aligned}
$$

Obviously, such symbols satisfy hypotheses (3.9) which guarantee an improvement of the performance with respect to the classical Schwarz algorithm.

Symbols (4.1) give operators $S_{1}$ and $S_{2}$ that are difficult to use numerically. Thus, we assume, in this first analysis, that the frequencies of the solution are concentrated close to $k=k_{0}$ and $m=m_{0}$, so that we can take symbols close to the above optimal choice, but easier to handle. 
4.1. Constant approximations for localized frequencies. From the optimal symbols (4.1), we easily obtain that suitable constant approximation values are given by evaluating them for $k=k_{0}$ and $m=m_{0}$, obtaining

$$
\begin{aligned}
& \sigma_{1, T 0}^{c y l}\left(m_{0}, k_{0}\right)=-\sqrt{k_{0}^{2}+\eta} \frac{K_{m_{0}}^{\prime}\left(\sqrt{k_{0}^{2}+\eta} b\right)}{K_{m_{0}}\left(\sqrt{k_{0}^{2}+\eta} b\right)}, \\
& \sigma_{2, T 0}^{c y l}\left(m_{0}, k_{0}\right)=-\sqrt{k_{0}^{2}+\eta} \frac{I_{m_{0}}^{\prime}\left(\sqrt{k_{0}^{2}+\eta} a\right)}{I_{m_{0}}\left(\sqrt{k_{0}^{2}+\eta} a\right)} .
\end{aligned}
$$

It is reasonable to think that this choice of symbols gives a faster convergence for initial data of the form $g(\varphi, z)=y(z) e^{i m_{0} \varphi}$, where $y$ has frequencies close to $k_{0}$. Indeed, notice that

$$
\widehat{g}(m, k)=\left\{\begin{array}{cc}
0 & \text { if } m \neq m_{0} \\
2 \pi \widehat{y}(k) & \text { if } m=m_{0}
\end{array}\right.
$$

The corresponding Optimized Schwarz Method has the following convergence factor

$$
\begin{gathered}
\rho_{T 0}^{c y l}\left(m, k, m_{0}, k_{0}\right)=\frac{-\sqrt{k_{0}^{2}+\eta} \frac{I_{m_{0}}^{\prime}\left(\sqrt{k_{0}^{2}+\eta} a\right)}{I_{m_{0}}\left(\sqrt{k_{0}^{2}+\eta} a\right)} I_{m}(\alpha a)+\alpha I_{m}^{\prime}(\alpha a)}{-\sqrt{k_{0}^{2}+\eta} \frac{I_{m_{0}}^{\prime}\left(\sqrt{k_{0}^{2}+\eta} a\right)}{I_{m_{0}}\left(\sqrt{k_{0}^{2}+\eta} a\right)} K_{m}(\alpha a)+\alpha K_{m}^{\prime}(\alpha a)} \times \\
\times-\frac{-\sqrt{k_{0}^{2}+\eta} \frac{K_{m_{0}}^{\prime}\left(\sqrt{k_{0}^{2}+\eta} b\right)}{K_{m_{0}}\left(\sqrt{k_{0}^{2}+\eta} b\right)} K_{m}(\alpha b)+\alpha K_{m}^{\prime}(\alpha b)}{-\sqrt{k_{0}^{2}+\eta} \frac{K_{m_{0}}^{\prime}\left(\sqrt{k_{0}^{2}+\eta} b\right)}{K_{m_{0}}\left(\sqrt{k_{0}^{2}+\eta} b\right)} I_{m}(\alpha b)+\alpha I_{m}^{\prime}(\alpha b)} .
\end{gathered}
$$

REMARK 2. The constant optimal values (4.2) are different from those derived by the "flat" analysis, that is

$$
\sigma_{1, T 0}^{\text {flat }}\left(s_{0}, k_{0}\right)=\sqrt{\left(s_{0}^{2}+k_{0}^{2}\right)+\eta}, \quad \sigma_{2, T 0}^{\text {flat }}\left(s_{0}, k_{0}\right)=-\sqrt{\left(s_{0}^{2}+k_{0}^{2}\right)+\eta},
$$

for suitable $s_{0}$ and $k_{0}$. Then, the hope is that the new constant optimized values (4.2) improve the convergence in those cases characterized by curve interfaces and by a cylindrical symmetry.

We observe that, as for the flat case, after discretization the maximum frequency $k$ is $\pi / h$, where $h$ is the space discretization parameter [8]. Assume that the overlap in the classical Schwarz method is $b-a=O(h)$. We have then the following result.

Proposition 5. Suppose that the solution is independent of $\varphi$, so that its only non-vanishing frequencies are those with $m=0$. Then the reduction factor (3.3) of the classical Schwarz method, as h goes to zero, has the following asymptotic behaviour

$$
\max _{|k| \leq k_{\max }}\left|\rho_{c l a}^{c y l}(0, k)\right|=\left|\rho_{c l a}^{c y l}(0,0)\right|=1-\sqrt{\eta}\left(\frac{K_{1}(\sqrt{\eta} a)}{K_{0}(\sqrt{\eta} a)}+\frac{I_{1}(\sqrt{\eta} a)}{I_{0}(\sqrt{\eta} a)}\right) h+O\left(h^{2}\right) .
$$

As for the Optimized Schwarz Method with constant approximations of the symbols in the transmission conditions and without overlap, the reduction factor (4.3) computed for $k_{0}=m_{0}=0$, as $h$ goes to zero, behaves as

$\max _{|k| \leq k_{\max }}\left|\rho_{T 0}^{c y l}(0, k, 0,0)\right|=\left|\rho_{T 0}^{c y l}\left(0, k_{\max }, 0,0\right)\right|=1-\frac{2 \sqrt{\eta}}{\pi}\left(\frac{K_{1}(\sqrt{\eta} a)}{K_{0}(\sqrt{\eta} a)}+\frac{I_{1}(\sqrt{\eta} a)}{I_{0}(\sqrt{\eta} a)}\right) h+O\left(h^{2}\right)$. 
Proof. As for the first result, we start from (3.3) and we evaluate it for $m=0$, obtaining

$$
\rho_{c l a}^{c y l}(0, k)=\frac{I_{0}(\alpha a)}{I_{0}(\alpha b)} \frac{K_{0}(\alpha b)}{K_{0}(\alpha a)}
$$

We consider the first factor and we study when it is a decreasing function of $k \geq 0$. This happens when its derivative with respect to $\alpha$ is less than zero (remember that $\left.\alpha=\sqrt{k^{2}+\eta}\right)$, that is when

$$
\frac{a I_{o}^{\prime}(\alpha a) I_{o}(\alpha b)-b I_{o}(\alpha a) I_{o}^{\prime}(\alpha b)}{I_{0}^{2}(\alpha b)}<0 .
$$

This leads to condition

$$
\frac{a I_{0}^{\prime}(\alpha a)}{I_{0}(\alpha a)}<\frac{b I_{0}^{\prime}(\alpha b)}{I_{0}(\alpha b)}
$$

(remember that $I_{0}(x)>0$ for any $x \geq 0$ ). The latter condition happens if and only if the function $\psi(z):=z I_{0}^{\prime}(z) / I_{0}(z)$ is increasing. Taking the derivative of $\psi$ we obtain condition

$$
\frac{\left(I_{0}^{\prime}(z)+z I_{0}^{\prime \prime}(z)\right) I_{0}(z)-z\left(I_{0}^{\prime}(z)\right)^{2}}{I_{0}^{2}(z)}>0 .
$$

Recalling that $I_{0}$ satisfies the modified Bessel equation (5.1)

$$
u^{\prime \prime}+\frac{1}{z} u^{\prime}-u=0
$$

we have

$$
z I_{0}(z)^{\prime \prime}+I_{0}^{\prime}(z)=z I_{0}(z)
$$

which, substituted in condition (4.7), gives

$$
z\left(1-\left(\frac{I_{0}^{\prime}(z)}{I_{0}(z)}\right)^{2}\right)>0
$$

Since $0 \leq I_{0}^{\prime}(z)=I_{1}(z)<I_{0}(z)$, we have that the last inequality is always satisfied, and we conclude that the first factor in (4.6) is a decreasing function of $\alpha$ (and then of $k$ ). A similar argument holds for the second factor, so that we obtain that the reduction factor (4.6) is a decreasing function of $k \geq 0$. Therefore, it attains its maximum for $k=0$. Then, by evaluating (4.6) for $k=0$ and for $b=a+h$ and by using (5.3), the Taylor expansion for $h$ small leads to (4.5).

As for the second result, we start from (4.3) with $a=b$ and we compute it for $k_{0}=m_{0}=0$. By evaluating this function for $m=0$, we obtain thanks to (5.3)

$$
\rho_{T 0}^{c y l}(0, k, 0,0)=\frac{-\widetilde{\sigma}_{2} I_{0}(a \alpha)+\alpha I_{1}(a \alpha)}{\widetilde{\sigma}_{1} I_{0}(a \alpha)+\alpha I_{1}(a \alpha)} \frac{-\widetilde{\sigma}_{1} K_{0}(a \alpha)+\alpha K_{1}(a \alpha)}{\widetilde{\sigma}_{2} K_{0}(a \alpha)+\alpha K_{1}(a \alpha)},
$$


where $\widetilde{\sigma}_{1}=\sigma_{1, T 0}^{c y l}(0,0)=-\sqrt{\eta} \frac{K_{0}^{\prime}(a \sqrt{\eta})}{K_{0}(a \sqrt{\eta})}>0$ and $\widetilde{\sigma}_{2}=-\sigma_{2, T 0}^{c y l}(0,0)=\sqrt{\eta} \frac{I_{0}^{\prime}(a \sqrt{\eta})}{I_{0}(a \sqrt{\eta})}>0$ (see $(4.2))$. It is possible to see that function $\rho_{T 0}^{c y l}(0, k, 0,0)$ is increasing in $k$. Indeed, observe that the derivative with respect to $\alpha$ of the first factor

$$
\frac{d}{d \alpha}\left(\frac{-\widetilde{\sigma}_{2} I_{0}(a \alpha)+\alpha I_{1}(a \alpha)}{\widetilde{\sigma}_{1} I_{0}(a \alpha)+\alpha I_{1}(a \alpha)}\right)=\frac{\left(\widetilde{\sigma}_{1}+\hat{\sigma}_{2}\right)\left(I_{0}(a \alpha)\right)^{2}}{\left(\widetilde{\sigma}_{1} I_{0}(a \alpha)+\alpha I_{1}(a \alpha)\right)^{2}} \frac{d}{d \alpha}\left(\frac{\alpha I_{1}(a \alpha)}{I_{0}(a \alpha)}\right)
$$

is positive, since the function $\frac{\alpha I_{1}(a \alpha)}{I_{0}(a \alpha)}=\frac{\alpha I_{0}^{\prime}(a \alpha)}{I_{0}(a \alpha)}$ is increasing in $\alpha$, as proved above. A similar argument shows that the second factor in (4.8) is increasing in $\alpha$ too. It follows that the maximum of $\rho_{T 0}^{c y l}(0, k, 0,0)$ is reached for $k=k_{\text {max }}=\pi / h$. When $h$ is small, then $\alpha_{\max }:=\sqrt{\eta+k_{\max }^{2}}=\sqrt{\eta+\pi^{2} / h^{2}}=\pi / h+O(h)$ is large and, from the asymptotic behaviour near infinity of the modified Bessel functions $[1,15]$

$$
\begin{aligned}
I_{m}(x) & =\frac{e^{x}}{\sqrt{2 \pi x}}\left(1-\frac{4 m^{2}-1}{8 x}+O\left(\frac{1}{x^{2}}\right)\right), \\
K_{m}(x) & =\sqrt{\frac{\pi}{2 x}} e^{-x}\left(1+\frac{4 m^{2}-1}{8 x}+O\left(\frac{1}{x^{2}}\right)\right),
\end{aligned}
$$

as $x \rightarrow+\infty$, it follows that

$$
\begin{aligned}
& \max _{|k| \leq k_{\max }}\left|\rho_{T 0}^{c y l}(0, k, 0,0)\right|=\left|\rho_{T 0}^{c y l}\left(0, k_{\max }, 0,0\right)\right|= \\
&=\left|\frac{-\widetilde{\sigma}_{2}\left(1+\frac{1}{8 a \alpha_{\max }}+O\left(\frac{1}{\alpha_{\max }^{2}}\right)\right)+\alpha_{\max }\left(1-\frac{3}{8 a \alpha_{\max }}+O\left(\frac{1}{\alpha_{\max }^{2}}\right)\right)}{\widetilde{\sigma}_{1}\left(1+\frac{1}{8 a \alpha_{\max }}+O\left(\frac{1}{\alpha_{\max }^{2}}\right)\right)+\alpha_{\max }\left(1-\frac{3}{8 a \alpha_{\max }}+O\left(\frac{1}{\alpha_{\max }^{2}}\right)\right)}\right| \times \\
& \times\left|\frac{-\widetilde{\sigma}_{1}\left(1-\frac{1}{8 a \alpha_{\max }}+O\left(\frac{1}{\alpha_{\max }^{2}}\right)\right)+\alpha_{\max }\left(1+\frac{3}{8 a \alpha_{\max }}+O\left(\frac{1}{\alpha_{\max }^{2}}\right)\right)}{\widetilde{\sigma}_{2}\left(1-\frac{1}{8 a \alpha_{\max }}+O\left(\frac{1}{\alpha_{\max }^{2}}\right)\right)+\alpha_{\max }\left(1+\frac{3}{8 a \alpha_{\max }}+O\left(\frac{1}{\alpha_{\max }^{2}}\right)\right)}\right|= \\
&=1-\frac{2\left(\widetilde{\sigma}_{1}+\widetilde{\sigma}_{2}\right)}{\alpha_{\max }}+O\left(\frac{1}{\alpha_{\max }^{2}}\right)= \\
&=1-\frac{2\left(\widetilde{\sigma}_{1}+\widetilde{\sigma}_{2}\right)}{\pi} h+O\left(h^{2}\right), \text { as } h \rightarrow 0,
\end{aligned}
$$

and the thesis follows.

The previous result shows that the asymptotic performance of the classical Schwarz method with overlap of the order of the space discretization parameter is the same of the generalized Schwarz method with optimized constant parameters in the transmission conditions without overlap. This result could be seen as the counterpart for cylindrical interfaces of that obtained for the flat case (see Theorem 4.2 in [8]).

4.2. Second order approximations. We look here for optimal second order approximations of type

$$
\sigma_{1, a p p}^{c y l}=p_{1}+q_{1} k^{2}, \quad \sigma_{2, a p p}^{c y l}=-p_{2}-q_{2} k^{2},
$$

for $p_{1}, p_{2}, q_{1}, q_{2} \in \mathbb{R}^{+}$. Such approximations come out for example from the Taylor expansion with respect to $k$ of $\sigma_{1, o p t}^{c y l}\left(m_{0}, k\right)$ and $\sigma_{2, \text { opt }}^{c y l}\left(m_{0}, k\right)$ in $(4.1)$ to the second order and centered in $k=k_{0}$. For simplicity reasons, let us take $k_{0}=m_{0}=0$. We 
obtain the following values (see the Appendix for the calculations)

$$
\begin{aligned}
& \sigma_{1, T 2}^{c y l}(k)=\sqrt{\eta} \frac{K_{1}(\sqrt{\eta} b)}{K_{0}(\sqrt{\eta} b)}+\frac{b}{2}\left(\left(\frac{K_{1}(\sqrt{\eta} b)}{K_{0}(\sqrt{\eta} b)}\right)^{2}-1\right) k^{2}, \\
& \sigma_{2, T 2}^{c y l}(k)=-\sqrt{\eta} \frac{I_{1}(\sqrt{\eta} a)}{I_{0}(\sqrt{\eta} a)}-\frac{a}{2}\left(1-\left(\frac{I_{1}(\sqrt{\eta} a)}{I_{0}(\sqrt{\eta} a)}\right)^{2}\right) k^{2} .
\end{aligned}
$$

In Figures 4.1 we report the values of different reduction factors as a function of the frequency $k$, for $\eta=1, a=0.495$ and $b=0.5$. In particular, we have considered the reduction factor (3.3) for the classical Schwarz method and the reduction factor (4.3) for the generalized Schwarz method obtained with optimal constant choices of the interface symbols. We observe that quantity (4.3) depends on the frequencies $k_{0}$ and $m_{0}$ chosen to compute the constant optimal interface symbols. To highlight the dependence of such quantity on this choice, we considered three cases, namely $m_{0}=0$ (figures at top), $m_{0}=1$ (figure at middle), and $m_{0}=10$ (figure at bottom). Since we did not experience significant qualitative differences among different choices of $k_{0}$, in all the depicted figures we considered only the case $k_{0}=0$. For the upper case $\left(m_{0}=0\right)$ we considered also the reduction factor $(3.7)$ with symbols given by (4.9), corresponding to the optimal second order interface approximations. Moreover, we observe that the reduction factors (3.3) and (4.3) are functions of the discrete frequency $m$. For this reason, we considered, for each case, three values of $m$, namely $m=0$ (figures at the left), $m=1$ (figure at the middle), and $m=10$ (figures at the right).

From these figures we observe that in all the cases the classical Schwarz method is slower than the Optimized Schwarz Method, for any frequency $k$ and $m$. Figures at top highlighted also that the second order approximations (4.9) reduce significantly the reduction factor with respect to the constant approximations.

REMARK 3. In this work we considered second order optimized terms with respect to the variable $k$ solely. The investigation of second order approximations with respect to the variable $m$ too is under study, so that we do not consider this case here.

5. Numerical results. In this section we present some numerical results obtained by two 3D numerical experiments, aiming at validating the theoretical results of previous sections.

In all the simulations we have considered no overlap, we have denoted the common interface by $\Sigma$ and, if not otherwise specified, we have set $\eta=1$. As stopping criterion we considered

$$
\left\|u_{1}^{n}-u_{2}^{n}\right\|_{L^{2}(\Sigma)}+\left\|\frac{\partial u_{1}^{n}}{\partial r}-\frac{\partial u_{2}^{n}}{\partial r}\right\|_{L^{2}(\Sigma)}<\varepsilon
$$

where $\varepsilon=10^{-5}$ is the given tolerance and where the regularity of the forcing terms guarantees that the exact solution $u \in H^{2}(\Omega)$, so that all the norms in the criterion make sense.

All the numerical simulations have been performed by implementing the classical and the generalized Schwarz methods in the parallel Finite Element library LIFEV (www.lifev.org), developed at MOX, Politecnico di Milano, at CMCS, EPF de Lausanne, at INRIA in Paris, at Emory University. 

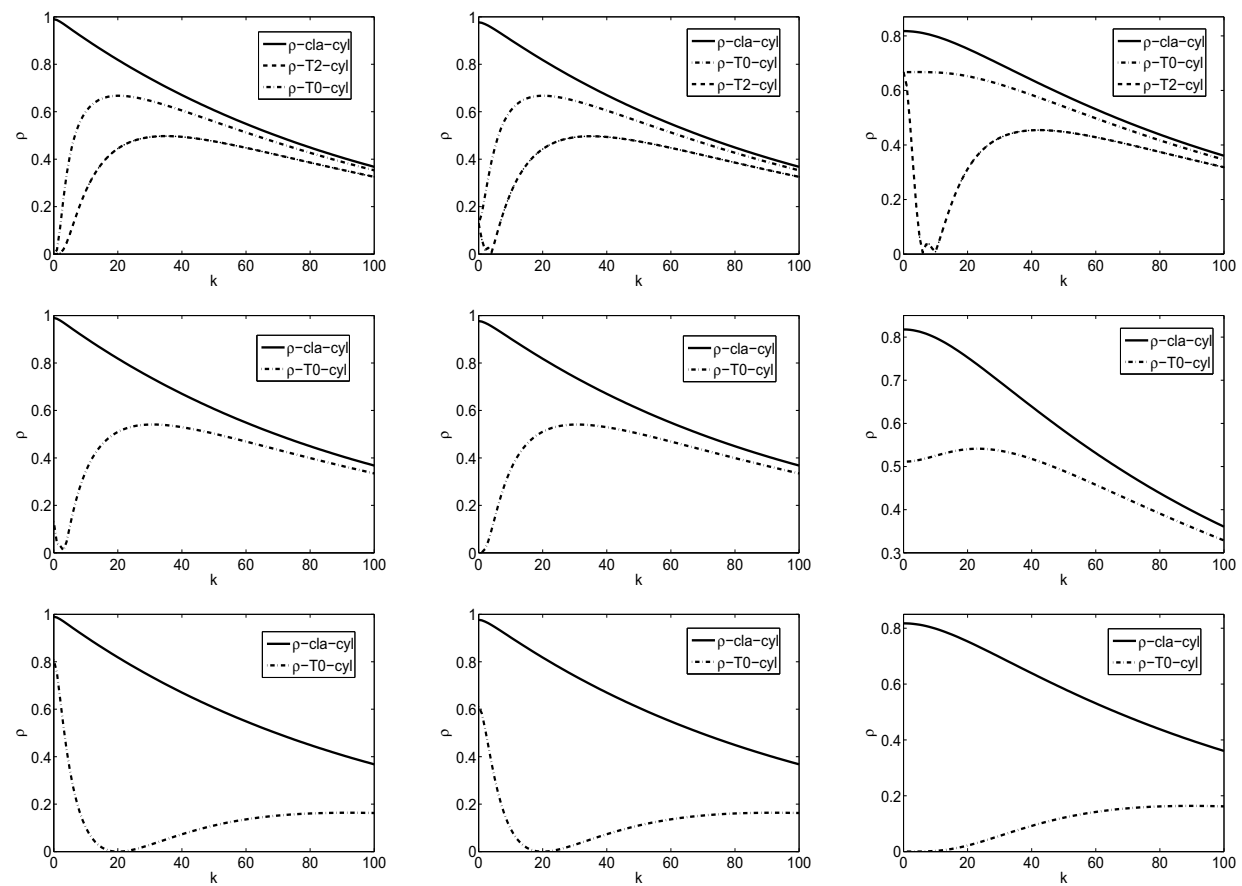

FIG. 4.1. Different reduction factors $\rho$ as a function of the frequency $k$. In all the cases $k_{0}=0$ has been considered. The reduction factor (4.3) has been computed for $m_{0}=0, u p, m_{0}=1$, middle, and $m_{0}=10$, bottom. For the discrete frequency we considered $m=0$ (left), $m=1$ (middle), and $m=10$ (right).

5.1. A test with flat interface. In the first numerical experiment, we want to investigate the convergence behaviour of the optimal values obtained in the analysis with cylindrical interfaces when applied to a case with a flat interface. To this aim, we considered as computational subdomains the two cubes $\Omega_{1}=[-0.5,0.5] \times[-0.5,0.5] \times$ $[0,1]$ and $\Omega_{2}=[-0.5,0.5] \times[-0.5,0.5] \times[1,2]$, with interface given by $\Sigma=\Omega_{1} \cap\{z=1\}$, see Figure 5.1, and we investigated the effectiveness of the constant optimal interface parameters (4.2) by varying the value of $a$. We set $f=0$, so that the exact solution is $u=0$, and then we evaluated the constant parameters (4.2) for $k_{0}=m_{0}=0$. As initial guess, we set

$$
\begin{cases}u_{2}^{0}=\left(\left(1-4 x^{2}\right)\left(1-4 y^{2}\right)\right)^{3} & \text { on } \Sigma, \\ \frac{\partial u_{2}^{0}}{\partial n}=0 & \text { on } \Sigma .\end{cases}
$$

In Table 5.1 we report the values of the constant parameters used in the numerical simulations for different values of $a$, and the related number of iterations needed to reach convergence. From these results, we observe that the best performance has been obtained for $a=+\infty$. This is not surprising, since in this test we are considering a flat interface, so that the best optimal constant interface parameters are supposed to be those obtained from the flat analysis. This is exactly the case $a=b=+\infty$, for which the optimal constant interface parameters (4.2) coincide with (4.4) for $s_{0}=0$.

5.2. A test with curve interface and cylindrical symmetry. In the second numerical test, we aimed at investigating the performance of the optimized parameters 


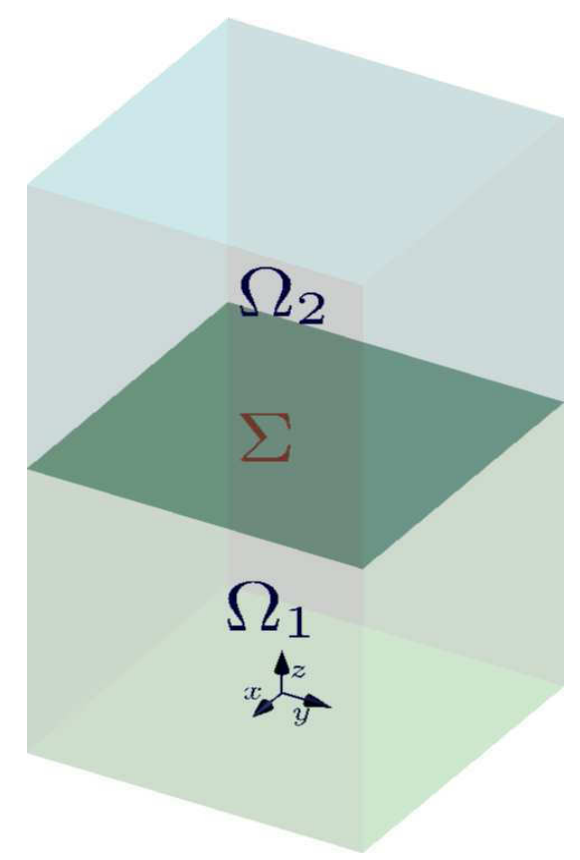

FIG. 5.1. Computational subdomains for the test with flat interface.

\begin{tabular}{c|cccc}
$\sigma / a$ & 0.1 & 1 & 10 & $+\infty$ \\
\hline \hline$\sigma_{1, T 0}^{c y l}$ & 4.06 & 1.43 & 1.05 & 1.00 \\
$\sigma_{2, T 0}^{c y l}$ & -0.05 & -0.45 & -0.95 & -1.00 \\
\hline$\#$ iter & 203 & 91 & 86 & 81
\end{tabular}

Values of the constant interface parameters for different values of $a . \eta=1$.

derived by the cylindrical analysis for a case characterized by a cylindrical symmetry and without overlap. In particular, the computational subdomains are given by $\Omega_{1}=$ $(r, \varphi, z)=[0, a] \times[0,2 \pi) \times[0, L]$ and $\Omega_{2}=(r, \varphi, z)=[a, R] \times[0,2 \pi) \times[0, L]$, with $a=0.5, L=5$ and $R=1$, see Figure 5.2. The values of the transmission conditions are the constant optimized values given by (4.4) and (4.2).

5.2.1. Varying $\eta$. In the first simulation, we set again $f=u=0$, and we investigated the performance of (4.4) and (4.2) with $k_{0}=s_{0}=m_{0}=0$ for different values of $\eta$.

In Table 5.2 we reported the constant optimized values used in the numerical simulations, while in Table 5.3 we reported the number of iterations needed to reach convergence. In Figure 5.3 we plotted the discrepancy at the interface between the traces and between the normal derivatives of $u$.

In this case, we observe a clear improvement in the convergence rate by choosing the parameters coming from the cylindrical analysis. In particular, the performance seems to improve for decreasing values of $\eta$. This shows the effectiveness of the optimization values obtained by the new analysis when a cylindrical symmetry characterizes the computational domain. 


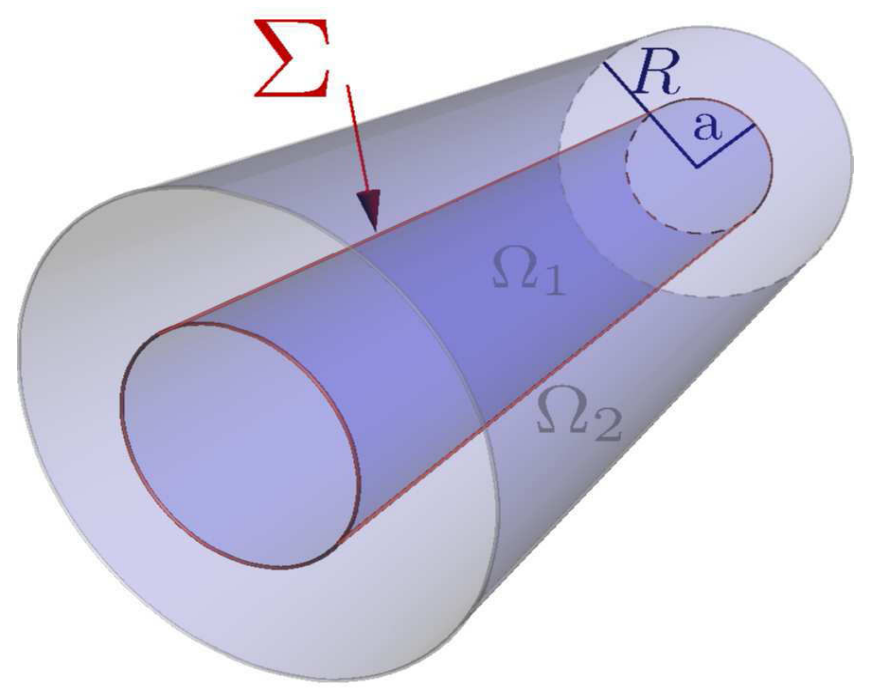

FIG. 5.2. Computational subdomains for the test with curve interface.

\begin{tabular}{c|c|c|c}
$\sigma / \eta$ & 0.1 & 1 & 10 \\
\hline \hline$\sigma_{1, T 0}^{\text {flat }}$ & 0.32 & 1.00 & 3.16 \\
$\sigma_{2, T 0}^{\text {flat }}$ & -0.32 & -1.00 & -3.16 \\
\hline$\sigma_{1, T 0}^{\text {cyl }}$ & 0.98 & 1.79 & 4.06 \\
$\sigma_{2, T 0}^{\text {cyl }}$ & -0.02 & -0.24 & -1.95 \\
\multicolumn{3}{|c}{ TABLE } & 5.2
\end{tabular}

Values of the constant optimized parameters used in the numerical experiments. Test with cylindrical interface.

\begin{tabular}{c||c|c|c}
$\sigma / \eta$ & 0.1 & 1 & 10 \\
\hline \hline$\sigma_{T 0}^{\text {flat }}$ & 160 & 92 & 55 \\
$\sigma_{T 0}^{\text {cyl }}$ & 28 & 35 & 39 \\
\multicolumn{2}{c}{ TABLE } & 5.3
\end{tabular}

Number of iterations for different values of $\eta$. Test with cylindrical interface.

5.2.2. Varying $a$. In the second experiment of this test, we want to study the sensitivity of the convergence on the ratio between $R$, the external radius, and $a$ the internal radius. This is due to the fact that we expect that the convergence improves by using the optimized parameters derived by the cylindrical analysis when this ratio goes to infinity, since such analysis has been performed for $R=\infty$.

In Table 5.4 we reported the number of iterations needed to reach convergence for three different values of the internal radius $a$ and using the constant optimized parameters $(4.2)$ with $k_{0}=m_{0}=0$.

As expected, the convergence properties of the generalized Schwarz method with constant parameters derived by the cylindrical analysis improved for decreasing values of $a$. 

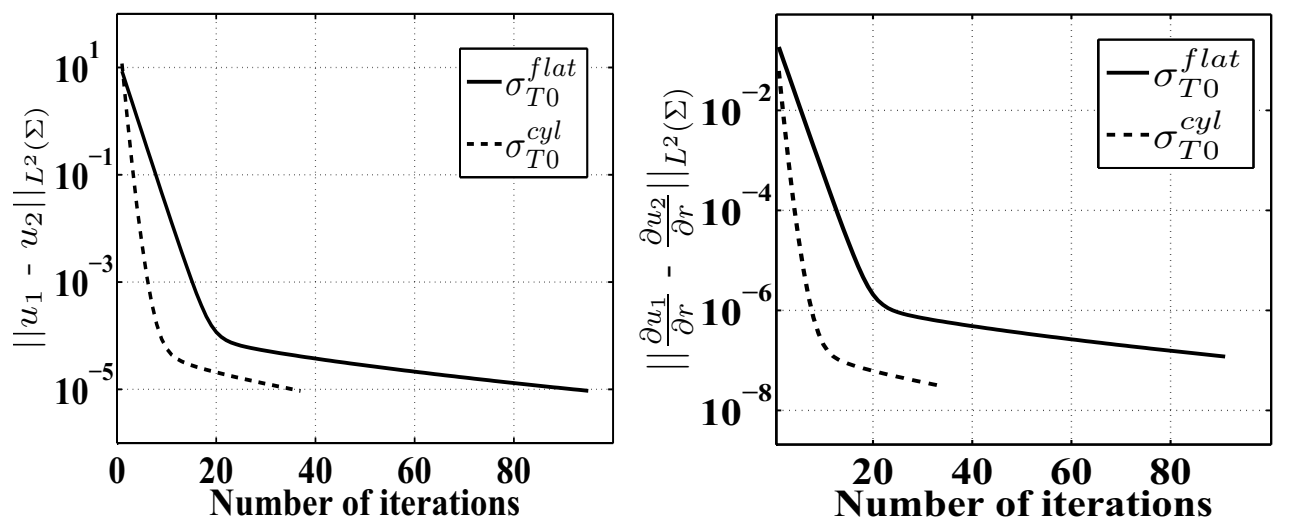

FIG. 5.3. Discrepancy at the interface between the traces (left) and between the normal derivatives (right) of $u$. Test with cylindrical interface.

\begin{tabular}{c||c|c|c} 
a & 0.1 & 0.5 & 0.9 \\
\hline \# iter & 30 & 35 & 66 \\
\cline { 2 - 2 } TABLE & 5.4
\end{tabular}

Number of iterations for different value of a. Test with cylindrical interface.

5.2.3. A case with a non-null localized angular frequency. In the third simulation of this test, we aimed at investigating the efficiency of the optimal constant interface parameters (4.2) when the solution features a dominant localized angular frequency $m_{0} \neq 0$, In particular, we considered as exact solution $u_{e x}=\left(x^{2}-y^{2}\right) z$, which is given by taking as right hand side $f=\eta u_{e x}$. This solution is characterized by a single angular frequency, namely $m_{0}=2$. As initial guess we took

$$
\begin{cases}u_{2}^{0}=5\left(x^{2}-y^{2}\right) \sin (\pi z / 5) & \text { on } \Sigma \\ \frac{\partial u_{2}^{0}}{\partial n}=0 & \text { on } \Sigma .\end{cases}
$$

In Table 5.5 we reported the values of the optimized parameters used in the numerical simulations (left, with $\sigma(j)$ we meant that (4.2) has been computed for $m_{0}=j$ ), and the number of iterations needed to reach convergence (right).

\begin{tabular}{c||c|c||c} 
& $\sigma_{1}$ & $\sigma_{2}$ & num iter \\
\hline \hline$\sigma_{T 0}^{\text {flat }}$ & 1.00 & -1.00 & 284 \\
$\sigma_{T 0}^{\text {cyl }}(0)$ & 1.80 & -0.24 & 283 \\
$\sigma_{T 0}^{\text {cyl }}(2)$ & 4.22 & -4.08 & 60
\end{tabular}

Values of the constant parameters used in the numerical simulations (left) and number of iterations to reach convergence (right). Test with cylindrical interface with localized angular frequency $m_{0}=2$.

These results highlighted that the knowledge a priori of the dominant angular frequency featured by the solution is fundamental to obtain efficient constant parameters in the transmission conditions. In particular, the convergence has been improved of 

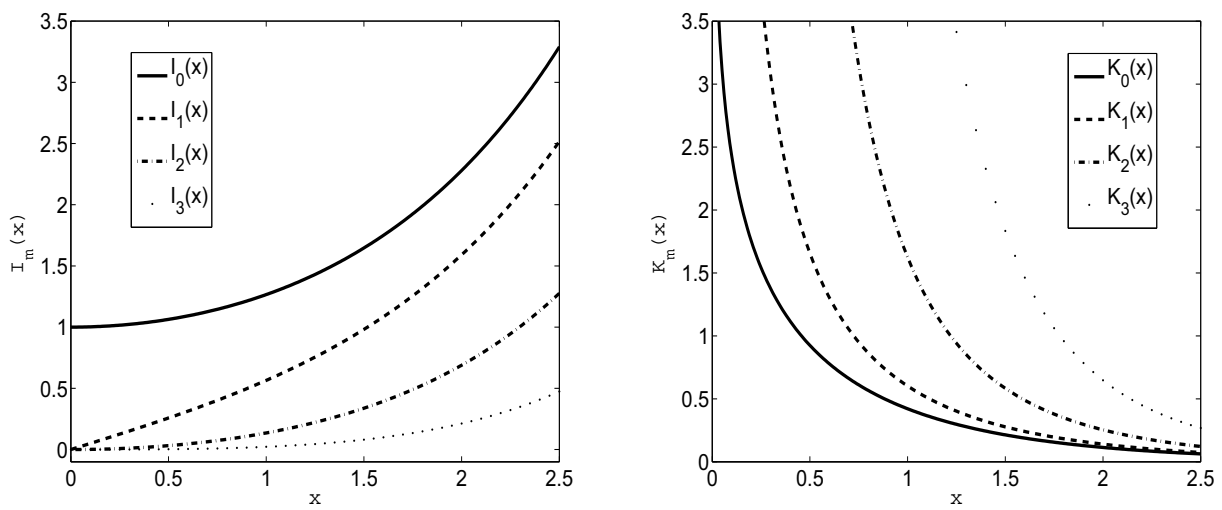

FIG. 5.4. Modified Bessel functions of first (left) and second (right) kind, $m=0,1,2,3$.

a factor 4 with respect both to the value coming from the flat analysis and to that obtained by the cylindrical analysis evaluated in $m_{0}=0$.

\section{Appendix.}

Notes on the modified Bessel functions. We give here some details regarding the modified Bessel's functions. For further details the interested readers could see $[1,15]$.

The modified Bessel's functions of first $I_{m}(x)$ and of second $K_{m}(x)$ kind are solutions to the modified Bessel's equation

$$
x^{2} \frac{d^{2} y}{d x^{2}}+x \frac{d y}{d x}-\left(x^{2}+m^{2}\right) y=0 .
$$

Their expressions for $m \in \mathbb{N}$, are given by

$$
\begin{aligned}
& I_{m}(x)=\sum_{j=0}^{\infty} \frac{(x / 2)^{m+2 j}}{j !(j+m) !} \\
& K_{m}(x)=-\frac{1}{2}\left(\frac{x}{2}\right)^{2} \int_{0}^{\infty} e^{-t-\left(x^{2} / 4 t\right)} t^{-1-m} d t .
\end{aligned}
$$

As shown by Figure 5.4, we observe that both $I_{m}$ and $K_{m}$ are non-negative functions. Moreover, $I_{m}$ are increasing functions of $x$, going to infinity for $x \rightarrow \infty$, while $K_{m}$ are decreasing functions of $x$, going to infinity for $x \rightarrow 0$ and going to zero for $x \rightarrow \infty$. We have the following property for a fixed $x>0: I_{m}(x)>I_{p}(x)$ and $K_{m}(x)<K_{p}(x)$, for $m>p$. We also recall the following identities

$$
\begin{aligned}
& I_{m}^{\prime}(x)=\frac{1}{2}\left(I_{m+1}(x)+I_{m-1}(x)\right)>0, \\
& K_{m}^{\prime}(x)=-\frac{1}{2}\left(K_{m+1}(x)+K_{m-1}(x)\right)<0, \\
& I_{m}(x)=I_{-m}(x) \\
& K_{m}(x)=K_{-m}(x)
\end{aligned}
$$

which lead in particular to

$$
I_{0}^{\prime}(x)=I_{1}(x), \quad K_{0}^{\prime}(x)=-K_{1}(x) .
$$


From $(5.2)_{1,2}$, it follows also that $I_{m}^{\prime}(x)<I_{m}^{\prime}(y)$ and $K_{m}^{\prime}(x)<K_{m}^{\prime}(y)$, for $x<y$ and for any $m$.

Another useful formula is the following [15]

$$
\frac{d}{d x}\left(x^{m} K_{m}(x)\right)=-x^{m} K_{m-1}(x)
$$

Computation of second order approximations in Section 4.2. Let us begin with $\sigma_{1, T 2}^{c y l}$. We have

$$
\sigma_{1, o p t}^{c y l}(0, k)=\alpha \frac{K_{1}(\alpha b)}{K_{0}(\alpha b)}
$$

Thus

$$
\begin{aligned}
\frac{\partial \sigma_{1, o p t}^{c y l}}{\partial k}(0, k) & =\frac{\partial}{\partial \alpha}\left(\alpha \frac{K_{1}(\alpha b)}{K_{0}(\alpha b)}\right) \alpha^{\prime}(k), \\
\frac{\partial^{2} \sigma_{1, o p t}^{c y l}}{\partial k^{2}}(0, k) & =\frac{\partial^{2}}{\partial \alpha^{2}}\left(\alpha \frac{K_{1}(\alpha b)}{K_{0}(\alpha b)}\right)\left(\alpha^{\prime}(k)\right)^{2}+\frac{\partial}{\partial \alpha}\left(\alpha \frac{K_{1}(\alpha b)}{K_{j}(\alpha b)}\right) \alpha^{\prime \prime}(k) .
\end{aligned}
$$

Notice that $\alpha(0)=\sqrt{\eta}, \alpha^{\prime}(0)=0$ and $\alpha^{\prime \prime}(0)=1 / \sqrt{\eta}$, and therefore

$$
\begin{aligned}
\frac{\partial \sigma_{1, o p t}^{c y l}}{\partial k}(0,0) & =0 \\
\frac{\partial^{2} \sigma_{1, o p t}^{c y l}}{\partial k^{2}}(0,0) & =\left.\frac{1}{\sqrt{\eta}} \frac{\partial}{\partial \alpha}\left(\alpha \frac{K_{1}(\alpha b)}{K_{0}(\alpha b)}\right)\right|_{\alpha=\sqrt{\eta}}\left(K_{0}(\alpha b)\right)^{2} \\
& =\frac{1}{\sqrt{\eta}}\left[\frac{K_{1}(\alpha b)}{K_{0}(\alpha b)}+\alpha b \frac{K_{1}^{\prime}(\alpha b) K_{0}(\alpha b)-K_{1}(\alpha b) K_{0}^{\prime}(\alpha b)}{\alpha=\sqrt{\eta}}\right]_{\alpha} \\
& =b\left(\left(\frac{K_{1}(\sqrt{\eta} b)}{K_{0}(\sqrt{\eta} b)}\right)^{2}-1\right),
\end{aligned}
$$

where in the last equality we have used relation (5.4) with $m=0$ and the first of (5.3). Thus, set

$$
\sigma_{1, T 2}^{c y l}(k)=\sqrt{\eta} \frac{K_{1}(\sqrt{\eta} b)}{K_{0}(\sqrt{\eta} b)}+\frac{b}{2}\left(\left(\frac{K_{1}(\sqrt{\eta} b)}{K_{0}(\sqrt{\eta} b)}\right)^{2}-1\right) k^{2} .
$$

Similarly, from

$$
\sigma_{2, o p t}^{c y l}(0, k)=\alpha \frac{I_{1}(\alpha a)}{I_{0}(\alpha a)} .
$$

we obtain

$$
\sigma_{2, T 2}^{c y l}(k)=-\sqrt{\eta} \frac{I_{1}(\sqrt{\eta} a)}{I_{0}(\sqrt{\eta} a)}-\frac{a}{2}\left(1-\left(\frac{I_{1}(\sqrt{\eta} a)}{I_{0}(\sqrt{\eta} a)}\right)^{2}\right) k^{2} .
$$




\section{REFERENCES}

[1] M. Abramowitz And I.A. Stegun, Handbook of Mathematical Functions with Formulas, Graphs, and Mathematical Tables, New York: Dover, 1965, pp. 1325-1328.

[2] P.F. Antonietti And B. Ayuso, Schwarz domain decomposition preconditioners for discontinuous Galerkin approximations of elliptic problems: non-overlapping case, M2AN Math. Model. Numer. Anal., 41 (2007), pp. 21-54.

[3] — Two-level Schwarz preconditioners for super penalty discontinuous Galerkin methods, Commun. Comput. Phys., 5 (2009), pp. 398-412.

[4] P.E. BJorstad AND O.B. Widlund, Iterative methods for the solution of elliptic problems on regions partitioned into substructures, SIAM Journal on Numerical Analysis, 23 (1986), pp. 1097-1120.

[5] P. Charton, F. Nataf, and F. Rogier, Méthode de décomposition de domain pour l'équation d'advection-diffusion, C.R. Acad. Sci., 313 (1991), pp. 623-626.

[6] V. Dolean, M.J. Gander, and L. Gerardo Giorda, Optimized Schwarz Methods for Maxwell's equations, SIAM Journal on Scientific Computing, 31(3) (2009), pp. 2193-2213.

[7] X. Feng and O.A. Karakashian, Two-level additive Schwarz methods for a discontinuous Galerkin approximation of second order elliptic problems, SIAM J. Numer. Anal., 39 (2001), pp. 1343-1365 (electronic).

[8] M.J. Gander, Optimized Schwarz Methods, SIAM Journal on Numerical Analysis, 44(2) (2006), pp. 699-731.

[9] M.J. GANDER AND L. HALPERN, Optimized Schwarz waveform relaxation methods for advection reaction diffusion problems, SIAM Journal on Numerical Analysis, 45(2) (2007), pp. 666697.

[10] M.J. Gander, L. Halpern, And C. Japhet, Optimized Schwarz algorithms for coupling convection and convection-diffusion problems, in Proceedings of the Thirteenth International Conference on Domain Decomposition Methods, 2001, pp. 253-260.

[11] M.J. Gander, F. Magoulès, And F.NATAF, Optimized Schwarz methods without overlap for the Helmholtz equation, SIAM Journal on Scientific Computing, 24 (2002), pp. 38-60.

[12] L. Gerardo Giorda, F. Nobile, and C. Vergara, Analysis and optimization of Robin-Robin partitioned procedures in fluid-structure interaction problems, SIAM Journal on Numerical Analysis, 48(6) (2010), pp. 2091-2116.

[13] C. JAPHET, Optimized Krylov-Ventcell method. Application to convection-diffusion problems, in Proceedings of the Ninth International Conference on Domain Decomposition Methods, P.E. Bjorstad, M.S. Espedal, and D.E. Keyes, eds., 1998, pp. 382-389.

[14] C. Japhet, N. Nataf, and F. Rogier, The optimized order 2 method. Application to convection-diffusion problems, Fut Gen COmput Syst, 18 (2001), pp. 17-30.

[15] N. Lebedev, Special Functions and Their Applications, Courier Dover Publications, 1972.

[16] P.L. Lions, On the Schwartz alternating method iii, in Proceedings of the Third International Symposium on Domain Decomposition Methods for PDE's, T. Chan, R. Glowinki, J. Periaux, and O.B. Widlund, eds., Siam, Philadelphia, 1990, pp. 202-223.

[17] Y. MADAY AND F. MAGOUlÈs, Optimized Schwarz methods without overlap for hughly heterogeneous media, Computer Methods in Applied Mechanics and Engineering, 196 (2007), pp. 1541-1553.

[18] F. Magoulès, P. Ivanyi, And B.H.V. Topping, Non-overlapping Schwarz method with optimized transmission conditions for the Helmholtz equation, Computer Methods in Applied Mechanics and Engineering, 193 (2004), pp. 4797-4818.

[19] F. NATAF AND F. Rogier, Factorization of the convection-diffusion operator and the Schwarz algorithm, Mathematical Models and Methods in Applied Sciences, 5 (1995), pp. 67-93.

[20] F. Nobile, M. Pozzoli, And C. Vergara, Time accurate partitioned algorithms for the solution of fluid-structure interaction problems in haemodynamics, MOX-Report n. 30-2011, Department of Mathematics, Politecnico di Milano, Italy, 2011.

[21] A. Qaddouria, L. Laayounib, S. Loiselc, J Cotea, and M.J. Gander, Optimized Schwarz methods with an overset grid for the shallow-water equations: preliminary results, Appl. Num. Math, 58 (2008), pp. 459-471.

[22] A. Quarteroni and A. Valli, Domain Decomposition Methods for Partial Differential Equations, Oxford Science Publications, 1999.

[23] B. Stupfel, Improved transmission conditions for a one-dimensional domain decomposition method applied to the solution of the Helmhotz equation, Journal of Computational Physics, 229 (2010), pp. 851-874.

[24] J. XU, Iterative methods by space decomposition and subspace correction, SIAM Review, 34 (1992), pp. 581-613. 